MODELING, IDENTIFICATION AND CONTROL, 1989, vol. 10, NO. 1, 13-34

doi:10.4173/mic.1989.1.2

\title{
Convergence analysis of some decentralized parameter estimators
}

\author{
R. HENRIKSEN†
}

Keywords: decentralized estimation, least squares method, instrumental variable methods, bootstrap estimators, convergence analysis, adaptive control.

Parameter estimators based upon standard estimation techniques, viz. least squares (LS) methods, instrumental variable methods, etc. may occasionally have difficulties with systems having a somewhat ill-conditioned nature, e.g., stiff systems. The robustness of the estimator can, however, be significantly improved by employing some kind of decentralized estimation techniques. The method presented here utilizes certain moving average filters and a system of parallel estimators, where the moving average filters are used to prefilter the input-output data. The form of these filters depends upon the estimated model, and the decentralized estimator will therefore usually have to be employed in a bootstrap fashion. The convergence properties of this bootstrap estimator are analysed, and necessary and sufficient conditions for local convergence of some decentralized estimators based upon LS or IV techniques are derived.

\section{Introduction}

Parameter estimators based upon standard estimation techniques, viz. least squares (LS) methods, instrumental variable (IV) methods, etc. do occasionally have difficulties with systems that have a somewhat ill-conditioned nature, e.g. stiff systems. This is a particularly severe drawback with many self-tuning and adaptive controllers. For example, small variations in the estimated plant parameters can cause the process gain to change by orders of magnitude and even change sign. The same variations can also cause poles of the estimated plant to appear as complex conjugate pairs even though they actually are distinct and strictly real. An example of this can be found in the papers by Young et al. (1987) and Henriksen (1988).

By employing certain decentralized estimation techniques it is, however, possible to improve the robustness of the estimator significantly. The method presented in this paper is based upon the fact that if part of the dynamics of the plant was known, one could, by carefully filtering the output and/or the input, actually 'filter out' this known part of the plant model and end up with a reduced-order model. This approach is used by Clary et al. (1984) to remove the known dynamics and estimate only the unknown dynamics. In this paper the entire plant model will be considered to be unknown. The estimator is split up into smaller sub-estimators which are fed with properly filtered input-output data, enabling us to estimate the entire model in a decentralized fashion. This paper will be restricted to the case of two sub-estimators.

Received 1 August 1988.

$\dagger$ The Norwegian Institute of Technology, Division of Engineering Cybernetics, N-7034 Trondheim, Norway.

This research was carried out while the author visited the Department of Chemical and Nuclear Engineering, University of California, Santa Barbara, California. 
The filters used to filter the input-output data depend upon the estimated model. Since the latter generally differs from the true model beforehand, the proposed decentralized estimator will usually have to be employed in a bootstrap fashion. The main topic of this paper is the convergence properties of this bootstrap estimator.

The paper is organized as follows. In $\S 2$ there is a brief outline of the system model being used and some of the main underlying assumptions. Section 3 presents the form of three different decentralized estimators based upon LS techniques (one estimator) and IV techniques (two estimators) and shows how these estimators can be employed as bootstrap estimators. Section 4 is devoted to convergence analysis of these bootstrap estimators, and necessary and sufficient conditions for local convergence are derived for two of them. Some examples and comments on convergence rates etc. are presented in $\S 5$. A discussion of the results and proposals for future work are given in $\S 6$.

\section{System description}

Consider a system described by the discrete-time model

$$
A\left(q^{-1}\right) y_{t}=B\left(q^{-1}\right) u_{t}+v_{t}
$$

where $y_{t}$ is the output at time $t, u_{t}$ is the input, and $v_{t}$ is the disturbance or residual. The process $\left\{v_{t}\right\}$ is assumed to be a zero-mean stochastic process with a rational non-singular spectral density matrix (for the sake of simplicity we have occasionally assumed that $v_{t}=0$ for all $t$ ). The processes $\left\{u_{t}\right\}$ and $\left\{v_{t}\right\}$ will generally be assumed to be independent.

The matrix polynomials $A\left(q^{-1}\right)$ and $B\left(q^{-1}\right)$ are assumed to be of the form

$$
\begin{aligned}
& A\left(q^{-1}\right)=I+A_{1} q^{-1}+\ldots+A_{n_{A}}\left(q^{-n_{A}}\right) \\
& B\left(q^{-1}\right)=B_{1} q^{-1}+\ldots+B_{n_{B}}\left(q^{-n_{B}}\right)
\end{aligned}
$$

where $q^{-1}$ is the background shift operator. $A\left(q^{-1}\right)$ and $B\left(q^{-1}\right)$ are assumed to be left coprime. It is further assumed that the polynomial $A\left(q^{-1}\right)$ can be factored as

$$
A\left(q^{-1}\right)=A_{1}\left(q^{-1}\right) A_{2}\left(q^{-1}\right)=A_{2}\left(q^{-1}\right) A_{1}\left(q^{-1}\right)
$$

where

$$
\begin{aligned}
& A_{1}\left(q^{-1}\right)=I+A_{1}^{1} q^{-1}+\ldots+A_{n_{1}}^{1} q^{-n_{1}} \\
& A_{2}\left(q^{-1}\right)=I+A_{1}^{2} q^{-1}+\ldots+A_{n_{2}}^{2} q^{-n_{2}}
\end{aligned}
$$

and where $n_{1}+n_{2}=n_{1}$. The assumption that $A_{1}\left(q^{-1}\right)$ and $A_{2}\left(q^{-1}\right)$ commute (Eqn (4)) is of course limiting the use of the method which is about to be presented. It generally applies only to SISO (single-input single-output), MISO (multi-input single-output), and diagonal form MIMO (multi-input multi-output) models.

\section{Decentralized estimation of reduced-order models}

Assume the parameters in the polynomials $A_{2}\left(q^{-1}\right)$ and $B\left(q^{-1}\right)$ are all known. Defining the following moving average of the output $y_{t}$,

$$
w_{t}=A_{2}\left(q^{-1}\right) y_{t},
$$


we can rewrite (1) as (reduced-order model $M_{1}$ consisting of, e.g., the slow modes)

$$
A_{1}\left(q^{-1}\right) w_{t}=B\left(q^{-1}\right) u_{t}+v_{t}
$$

Now, in order to estimate the polynomial $A_{1}\left(q^{-1}\right)$ we could use a LS method (if $v_{t}$ is white), an IV method (if $v_{t}$ is coloured), etc. Sufficient conditions for consistency could be as follows: (1) the system described by the model (8) is linear, of finite order, and asymptotically stable; (2) the input process $\left\{u_{t}\right\}$ is stationary, ergodic with respect to second-order moments, and persistently exciting of order $n_{1} ;(3)$ the input $u_{t}$ and the disturbance $v_{s}$ are independent for all $t$ and $s ;(4)$ there is a parameter vector $\beta^{*}$ so that $A_{1}^{-1}\left(q^{-1}, \beta^{*}\right) B\left(q^{-1}\right) \equiv G_{1}\left(q^{-1}\right)$, where $G_{1}\left(q^{-1}\right)$ is the transfer function of the system described by $M_{1}$; and (5) the vector $\beta^{*}$ is unique, see Söderström and Stoica (1983). Note that these assumptions are not always necessary.

In a similar fashion, assume the parameters in the polynomial $A_{1}\left(q^{-1}\right)$ are all known. Defining the following moving average of the output $y_{t}$,

$$
z_{t}=A_{1}\left(q^{-1}\right) y_{t},
$$

we can rewrite (1) as (reduced-order model $M_{2}$ containing, e.g., the fast modes)

$$
A_{2}\left(q^{-1}\right) z_{t}=B\left(q^{-1}\right) u_{t}+v_{t}
$$

In order to estimate $A_{2}\left(q^{-1}\right)$ and $B\left(q^{-1}\right)$ we could again employ a LS method, an IV variant, etc. Sufficient conditions for consistency are similar to the ones given above. Note that $u_{t}$ in this case would have to be persistently exciting of order $n_{2}+n_{B}$.

Seemingly, in order to estimate both of the reduced-order models $M_{1}$ and $M_{2}$, it would suffice that $u_{t}$ is persistently exciting of order $\max \left(n_{1}, n_{2}+n_{B}\right)$. As we shall see, this is definitely not true.

\section{A Decentralized Least Squares (DLS) Estimator}

We now make the additional assumption that $v_{t}$ is white noise. The reducedorder model $M_{1}$ is rewritten as

$$
w_{t}=\Psi_{t}^{\mathrm{T}} \beta+B\left(q^{-1}\right) u_{t}+v_{t}
$$

where

$$
\begin{aligned}
\beta & =\left(\operatorname{row}\left[A_{1}^{1} A_{2}^{1} \ldots A_{n_{1}}^{1}\right]\right)^{\mathrm{T}} \\
\Psi_{t} & =\left[\begin{array}{cc}
\psi_{t} & 0 \\
\ddots & \\
0 & \psi_{t}
\end{array}\right]=I \otimes \psi_{t} \\
\psi_{t} & =\left[-w_{t-1}^{\mathrm{T}}, \ldots,-w_{t-n_{1}}^{\mathrm{T}}\right]^{\mathrm{T}}
\end{aligned}
$$

Here row $M$ denotes the row vector containing the rows of the matrix $\mathbf{M}$ in order, whereas $\otimes$ denotes the Kronecker matrix product, see Vetter $(1970,1973)$. The ordinary least squares estimate $\hat{\beta}$ of $\beta$ now takes the form

$$
\hat{\beta}=\left[(1 / N) \sum_{t=1}^{N} \Psi_{t} \Psi_{t}^{\mathrm{T}}\right]^{-1}\left[(1 / N) \sum_{t=1}^{N} \Psi_{t}\left(w_{t}-B\left(q^{-1}\right) u_{t}\right)\right]
$$

In a similar fashion we can rewrite the reduced-order model $M_{2}$ as

$$
z_{t}=\Phi_{t}^{\mathrm{T}} \theta+v_{t}
$$


where

$$
\begin{aligned}
\theta & =\left(\operatorname{row}\left[A_{1}^{2} A_{2}^{2} \ldots A_{n_{2}}^{2} B_{1} B_{2} \ldots B_{n_{B}}\right]\right)^{\mathrm{T}} \\
\Phi_{t} & =\left[\begin{array}{cc}
\varphi_{t} & 0 \\
\ddots & \\
0 & \varphi_{t}
\end{array}\right]=I \otimes \varphi_{t} \\
\varphi_{t} & =\left[-z_{t-1}^{\mathrm{T}}, \ldots,-z_{t-n_{2}}^{\mathrm{T}}, u_{t-1}^{\mathrm{T}}, \ldots, u_{t-n_{B}}^{\mathrm{T}}\right]^{\mathrm{T}}
\end{aligned}
$$

The ordinary least squares estimate $\hat{\theta}$ of $\theta$ takes the form

$$
\hat{\theta}=\left[(1 / N) \sum_{t=1}^{N} \Phi_{t} \Phi_{t}^{\mathrm{T}}\right]^{-1}\left[(1 / N) \sum_{t=1}^{N} \Phi_{t} z_{t}\right]
$$

Note that the two estimators given by $(11)-(15)$ and $(16)-(20)$ respectively do not have to use the same sampling rate. As a matter of fact, it may be advantageous to let them use different sampling rates. Transformation from a fast sampling rate to a slower one does not cause any difficulties although the term $B\left(q^{-1}\right) u_{t}$ appearing in (15) will only have a symbolic meaning; it is simply a known term representing the action of past and current inputs.

Both of the two estimators given above require that the output $y_{t}$ is filtered with the other reduced-order model's A-polynomial, i.e., $A_{1}\left(q^{-1}\right)$ and $A_{2}\left(q^{-1}\right)$ respectively. Since neither of these polynomials generally are known beforehand, we will have to use the pertinent estimated A-polynomial instead, i.e., $A_{1}\left(q^{-1}, \hat{\beta}\right)$ and $A_{2}\left(q^{-1}, \hat{\theta}\right)$ respectively. With this in mind, we now propose the following bootstrap estimator.

\section{Bootstrap algorithm (DLS Estimator)}

1. Start with $\hat{\beta}^{0}$ and $k:=0$.

2. Compute $z_{t}\left(\hat{\beta}^{k}\right)$ from

$$
z_{t}\left(\hat{\beta}^{k}\right)=A_{1}\left(q^{-1}, \hat{\beta}^{k}\right) y_{t}
$$

and $\hat{\theta}^{k+1}$ from

$$
\hat{\theta}^{k+1}=\left[(1 / N) \sum_{t=1}^{N} \Phi_{t}\left(\hat{\beta}^{k}\right) \Phi_{t}^{\mathrm{T}}\left(\hat{\beta}^{k}\right)\right]^{-1}\left[(1 / N) \sum_{t=1}^{N} \Phi_{t}\left(\hat{\beta}^{k}\right) z_{t}\left(\hat{\beta}^{k}\right)\right]
$$

3. Compute $w_{t}\left(\hat{\theta}^{k+1}\right)$ from

$$
w_{t}\left(\hat{\theta}^{k+1}\right)=A_{2}\left(q^{-1}, \hat{\theta}^{k+1}\right) y_{t}
$$

and $\hat{\beta}^{k+1}$ from

$$
\begin{aligned}
\hat{\beta}^{k+1}= & {\left[(1 / N) \sum_{t=1}^{N} \Psi_{t}\left(\hat{\theta}^{k+1}\right) \Psi_{t}^{\mathrm{T}}\left(\hat{\theta}^{k+1}\right)\right]^{-1} } \\
& \times\left[(1 / N) \sum_{t=1}^{N} \Psi_{t}\left(\hat{\theta}^{k+1}\right)\left[w_{t}\left(\hat{\theta}^{k+1}\right)-B\left(q^{-1}, \hat{\theta}^{k+1}\right) u_{t}\right]\right]
\end{aligned}
$$

4. If $k=0$ then $k:=1$ and return to 2 , else if $\hat{\beta}^{k+1} \neq \hat{\beta}^{k}$ or $\hat{\theta}^{k+1} \neq \hat{\theta}^{k}$ then $k:=k+1$ and return to 2 , else halt. 
The most crucial questions about the above bootstrap estimator are: (1) does it ever converge; and (2) if so, under what conditions does it converge. The convergence properties of this algorithm are the topic of the next section. To close this section we shall take a look at a couple of other estimators.

\section{Decentralized Instrumental Variable (DIV) Estimators}

If the process $\left\{v_{t}\right\}$ is coloured, we have to employ other methods for the design of our decentralized estimator. We shall now consider a couple of IV variants (for more details about IV methods, see Söderström and Stoica, 1983).

\section{DIV1 Estimator}

From the form of the DLS estimator we can directly derive the following IV variant

$$
\begin{aligned}
& \hat{\beta}=\left[(1 / N) \sum_{t=1}^{N} \tilde{\Psi}_{t} \Psi_{t}^{\mathrm{T}}\right]^{-1}\left[(1 / N) \sum_{t=1}^{N} \Psi_{t}\left(w_{t}-B\left(q^{-1}\right) u_{t}\right)\right] \\
& \hat{\theta}=\left[(1 / N) \sum_{t=1}^{N} \tilde{\Phi}_{t} \Phi_{t}^{\mathrm{T}}\right]^{-1}\left[(1 / N) \sum_{t=1}^{N} \tilde{\Phi}_{t} z_{t}\right]
\end{aligned}
$$

where

$$
\begin{aligned}
& \tilde{\Psi}_{t}=\left[\begin{array}{cc}
\tilde{\psi}_{t} & 0 \\
& \ddots \\
0 & \tilde{\psi}_{t}
\end{array}\right]=I \otimes \tilde{\psi}_{t} \\
& \tilde{\psi}_{t}=\left[-\tilde{w}_{t-1}^{\mathbf{T}}, \ldots,-\tilde{w}_{t-n_{1}}^{\mathbf{T}}\right]^{\mathbf{T}} \\
& \tilde{w}_{t}=A_{1}^{-1}\left(q^{-1}\right) B\left(q^{-1}\right) u_{t} \\
& \tilde{\Phi}_{t}=\left[\begin{array}{cc}
\tilde{\varphi}_{t} & 0 \\
& \ddots \\
0 & \tilde{\varphi}_{t}
\end{array}\right]=I \otimes \tilde{\varphi}_{t} \\
& \tilde{\varphi}_{t}=\left[-\tilde{z}_{t-1}^{\mathrm{T}}, \ldots,-\tilde{z}_{t-n_{2}}^{\mathrm{T}}, u_{t-1}^{\mathrm{T}}, \ldots, u_{t-n_{B}}^{\mathrm{T}}\right]^{\mathrm{T}} \\
& \tilde{z}_{t}=A_{2}^{-1}\left(q^{-1}\right) B\left(q^{-1}\right) u_{t}
\end{aligned}
$$

$\Psi_{t}$ and $\Phi_{t}$ are the same as previously.

\section{DIV2 Estimator}

This IV variant needs a little more explanation. Assume that $A_{2}\left(q^{-1}\right)$ and $B\left(q^{-1}\right)$ commute (generally this only applies to SISO and MISO systems). We now rewrite (1) as

$$
A_{1}\left(q^{-1}\right) y_{t}=B\left(q^{-1}\right) \tilde{u}_{t}+A_{2}^{-1}\left(q^{-1}\right) v_{t}
$$


where

$$
\tilde{u}_{t}=A_{2}^{-1}\left(q^{-1}\right) u_{t}
$$

From (33) we can derive the IV variant

$$
\hat{\beta}=\left[(1 / N) \sum_{t=1}^{N} \Psi_{t} \Psi_{t}^{\mathrm{T}}\right]^{-1}\left[(1 / N) \sum_{t=1}^{N} \Psi_{1}\left(y_{t}-B\left(q^{-1}\right) \tilde{u}_{t}\right)\right]
$$

where

$$
\begin{aligned}
\Psi_{t} & =I \otimes \psi_{t} ; \quad \tilde{\Psi}_{t}=I \otimes \tilde{\psi}_{t} \\
\psi_{t} & =\left[-y_{t-1}^{\mathrm{T}}, \ldots,-y_{t-n_{1}}^{\mathrm{T}}\right]^{\mathrm{T}} \\
\tilde{\psi} & =\left[-\tilde{y}_{t-1}^{\mathrm{T}}, \ldots,-\tilde{y}_{t-n_{1}}^{\mathrm{T}}\right]^{\mathrm{T}} \\
\tilde{y}_{t} & =A_{1}^{-1}\left(q^{-1}\right) B\left(q^{-1}\right) \tilde{u}_{t}
\end{aligned}
$$

In a similar fashion, assuming that $A_{1}\left(q^{-1}\right)$ and $B\left(q^{-1}\right)$ commute, (1) can be rewritten as

$$
A_{2}\left(q^{-1}\right) y_{t}=B\left(q^{-1}\right) \bar{u}_{t}+A_{1}^{-1}\left(q^{-1}\right) v_{t}
$$

where

$$
\bar{u}_{t}=A_{1}^{-1}\left(q^{-1}\right) u_{t}
$$

From (40) we derive the IV variant

$$
\hat{\theta}=\left[(1 / N) \sum_{t=1}^{N} \tilde{\Phi}_{t} \Phi_{t}^{\mathrm{T}}\right]^{-1}\left[(1 / N) \sum_{t=1}^{N} \Phi_{t} y_{t}\right]
$$

where

$$
\begin{aligned}
\Phi_{t} & =I \otimes \varphi_{t} ; \quad \tilde{\Phi}_{t}=I \otimes \tilde{\varphi}_{t} \\
\varphi_{t} & =\left[-y_{t-1}^{\mathrm{T}}, \ldots,-y_{t-n_{2}}^{\mathrm{T}}, \bar{u}_{t-1}^{\mathrm{T}}, \ldots, \bar{u}_{t-n B}^{\mathrm{T}}\right]^{\mathrm{T}} \\
\tilde{\varphi}_{t} & =\left[-\bar{y}_{t-1}^{\mathrm{T}}, \ldots,-\bar{y}_{t-n_{2}}^{\mathrm{T}}, \bar{u}_{t-1}^{\mathrm{T}}, \ldots, \bar{u}_{t-n B}^{\mathrm{T}}\right]^{\mathrm{T}} \\
\bar{y}_{t} & =A_{2}^{-1}\left(q^{-1}\right) B\left(q^{-1}\right) \bar{u}_{t}
\end{aligned}
$$

Note that $\psi_{t}, \varphi_{t}, \tilde{\psi}_{t}$, and $\tilde{\varphi}_{t}$ are all different from what they were in the DLS and DIV1 estimators. Also note that by the above assumptions,

$$
\tilde{y}_{t}=\bar{y}_{t}=\left[A_{1}\left(q^{-1}\right) A_{2}\left(q^{-1}\right)\right]^{-1} B\left(q^{-1}\right) u_{t}=A^{-1}\left(q^{-1}\right) B\left(q^{-1}\right) u_{t}
$$

These equations show that this estimator essentially is based upon filtering the input $u_{t}$ rather than the output $y_{t}$.

The bootstrap IV variants are similar to that given for the DLS estimator, but we have to start with $\hat{\beta}^{0}$ and $\hat{\theta}^{0}$ rather than only $\hat{\beta}^{0}$ (since $\tilde{z}_{t}$ now depends on $\hat{\theta}^{k}$, see (32) whereas $z_{t}$ depends on $\hat{\beta}^{k}$ ).

\section{Convergence analysis of the bootstrap estimators}

In addition to the assumptions made in the previous sections we now also assume that processes $\left\{u_{t}\right\}$ and $\left\{v_{t}\right\}$ are stationary and ergodic with respect to second order moments. 


\section{I. Analysis of the DLS Estimator}

From the assumptions above, as $N \rightarrow \infty$ the DLS estimator takes the form

$$
\begin{aligned}
& \hat{\beta}^{k+1}=\left[E \Psi_{t}\left(\hat{\theta}^{k+1}\right) \Psi_{t}^{\mathrm{T}}\left(\hat{\theta}^{k+1}\right)\right]^{-1} E \Psi_{t}\left(\hat{\theta}^{k+1}\right)\left[w_{t}\left(\hat{\theta}^{k+1}\right)-B\left(q^{-1}, \hat{\theta}^{k+1}\right) u_{t}\right] \\
& \hat{\theta}^{k+1}=\left[E \Phi_{t}\left(\hat{\beta}^{k}\right) \Phi_{t}^{\mathrm{T}}\left(\hat{\beta}^{k}\right)\right]^{-1} E \Phi_{t}\left(\hat{\beta}^{k}\right) z_{t}\left(\hat{\beta}^{k}\right)
\end{aligned}
$$

where $E$ is the expectation operator.

Let $\left(\beta^{*}, \theta^{*}\right)$ denote the true value of $(\beta, \theta)$. Note that if the consistency conditions in $\S 3$ are satisfied, then $\left(\beta^{*}, \theta^{*}\right)$ is indeed a convergence point. From (48) and (49) we obtain

$$
\begin{array}{r}
E \Psi_{t}\left(\theta^{*}\right)\left[w_{t}\left(\theta^{*}\right)-B\left(q^{-1}, \theta^{*}\right) u_{t}-\Psi_{t}^{\mathrm{T}}\left(\theta^{*}\right) \beta^{*}\right]=0 \\
E \Phi_{t}\left(\beta^{*}\right)\left[z_{t}\left(\beta^{*}\right)-\Phi_{t}^{\mathrm{T}}\left(\beta^{*}\right) \theta^{*}\right]=0
\end{array}
$$

By linearizing (48)-(49) about $\left(\beta^{*}, \theta^{*}\right)$ we find after some calculations

$$
\begin{aligned}
& \hat{\beta}^{k+1}-\beta^{*}=F_{1}\left(\theta^{*}\right)\left[\hat{\theta}^{k+1}-\theta^{*}\right] \\
& \hat{\theta}^{k+1}-\theta^{*}=F_{2}\left(\beta^{*}\right)\left[\hat{\beta}^{k}-\beta^{*}\right],
\end{aligned}
$$

that is,

$$
\hat{\beta}^{k+1}-\beta^{*}=F_{1}\left(\theta^{*}\right) F_{2}\left(\beta^{*}\right)\left[\hat{\beta}^{k}-\beta^{*}\right]
$$

or

$$
\hat{\theta}^{k+1}-\theta^{*}=F_{2}\left(\beta^{*}\right) F_{1}\left(\theta^{*}\right)\left[\hat{\theta}^{k}-\theta^{*}\right]
$$

where

$$
\begin{aligned}
F_{1}\left(\theta^{*}\right)= & M^{-1}\left(\theta^{*}\right)\left\{E \frac{\partial \Psi_{t}}{\partial \hat{\theta}^{\mathrm{T}}}\left(\theta^{*}\right)\left[I \otimes\left(w_{t}\left(\theta^{*}\right)-B\left(q^{-1}, \theta^{*}\right) u_{t}-\Psi_{t}^{\mathrm{T}}\left(\theta^{*}\right) \beta^{*}\right)\right]\right. \\
& +E \Psi_{t}\left(\theta^{*}\right)\left[\frac{\partial w_{t}}{\partial \hat{\theta}^{\mathrm{T}}}\left(\theta^{*}\right)-\frac{\partial B}{\partial \hat{\theta}^{\mathrm{T}}}\left(q^{-1}, \theta^{*}\right)\right. \\
& \left.\left.\times\left(I \otimes u_{t}\right)-\frac{\partial \Psi_{t}^{\mathrm{T}}}{\partial \hat{\theta}^{\mathrm{T}}}\left(\theta^{*}\right)\left(I \otimes \beta^{*}\right)\right]\right\} \\
F_{2}\left(\beta^{*}\right)= & L^{-1}\left(\beta^{*}\right)\left\{E \frac{\partial \Phi_{t}}{\partial \hat{\beta}^{\mathrm{T}}}\left(\beta^{*}\right)\left[I \otimes\left(z_{t}\left(\beta^{*}\right)-\Phi_{t}^{\mathrm{T}}\left(\beta^{*}\right) \theta^{*}\right)\right]\right. \\
& \left.+E \Phi_{t}\left(\beta^{*}\right)\left[\frac{\partial z_{t}}{\partial \hat{\beta}^{\mathrm{T}}}\left(\beta^{*}\right)-\frac{\partial \Phi_{t}^{\mathrm{T}}}{\partial \hat{\beta}^{\mathrm{T}}}\left(\beta^{*}\right)\left(I \otimes \theta^{*}\right)\right]\right\} \\
M\left(\theta^{*}\right)= & E \Psi_{t}\left(\theta^{*}\right) \Psi_{t}^{\mathrm{T}}\left(\theta^{*}\right) \\
L\left(\beta^{*}\right)= & E \Phi_{t}\left(\beta^{*}\right) \Phi_{t}^{\mathrm{T}}\left(\beta^{*}\right)
\end{aligned}
$$

see also Vetter $(1970,1973)$.

The DLS estimator converges locally if and only if the matrix

$$
\boldsymbol{F}\left(\beta^{*}, \theta^{*}\right)=\boldsymbol{F}_{1}\left(\theta^{*}\right) \boldsymbol{F}_{2}\left(\beta^{*}\right)
$$

(or $F^{\prime}\left(\beta^{*}, \theta^{*}\right)=F_{2}\left(\beta^{*}\right) F_{1}\left(\theta^{*}\right)$ ) has all of its eigenvalues strictly inside the unit circle. Note that the matrices $\boldsymbol{F}\left(\beta^{*}, \theta^{*}\right)$ and $\boldsymbol{F}^{\prime}\left(\beta^{*}, \theta^{*}\right)$ have exactly the same nonzero eigenvalues. We shall use the matrix $\boldsymbol{F}\left(\beta^{*}, \theta^{*}\right)$ in the remainder. 
The above expressions for $F_{1}\left(\theta^{*}\right)$ and $F_{2}\left(\beta^{*}\right)$ are quite general. By using the fact that $u_{t}$ and $v_{t}$ are independent and that $v_{t}$ is white, we find after some further calculations

$$
\begin{aligned}
& F_{1}\left(\theta^{*}\right)=-\left[E \Psi_{t}\left(\theta^{*}\right) \Psi_{t}^{\mathrm{T}}\left(\theta^{*}\right)\right]^{-1} E \Psi_{t}\left(\theta^{*}\right) \bar{\Phi}_{t}^{\mathrm{T}}\left(\beta^{*}\right) \\
& F_{2}\left(\beta^{*}\right)=-\left[E \Phi_{t}\left(\beta^{*}\right) \Phi_{t}^{\mathrm{T}}\left(\beta^{*}\right)\right]^{-1} E \Phi_{t}\left(\beta^{*}\right) \bar{\Psi}_{t}^{\mathrm{T}}\left(\theta^{*}\right)
\end{aligned}
$$

where

$$
\begin{aligned}
\bar{\Phi}_{t}\left(\beta^{*}\right) & =I \otimes \bar{\varphi}_{t}\left(\beta^{*}\right) ; \quad \bar{\Psi}_{t}\left(\theta^{*}\right)=I \otimes \bar{\psi}_{t}\left(\theta^{*}\right) \\
\bar{\varphi}_{t}\left(\beta^{*}\right) & =\left[-\bar{z}_{t-1}^{\mathrm{T}}, \ldots,-\bar{z}_{t-n_{2}}^{\mathrm{T}}, u_{t-1}^{\mathrm{T}}, \ldots, u_{t-n_{B}}^{\mathrm{T}}\right]^{\mathrm{T}} \\
\bar{\psi}_{t}\left(\theta^{*}\right) & =\left[-\bar{w}_{t-1}^{\mathrm{T}}, \ldots,-\bar{w}_{t-n_{1}}^{\mathrm{T}}\right]^{\mathrm{T}} \\
\bar{z}_{t} & =A_{1}^{\mathrm{T}}\left(q^{-1}, \beta^{*}\right) y_{t} ; \quad \bar{w}_{t}=A_{2}^{\mathrm{T}}\left(q^{-1}, \theta^{*}\right) y_{t}
\end{aligned}
$$

We have taken the effort of carrying this out for a somewhat general multivariable case just to see how far the analysis can be extended. It turns out that some very neat and useful results can be obtained if we assume $A\left(q^{-1}\right)=A_{1}\left(q^{-1}\right) A_{2}\left(q^{-1}\right)$ to be symmetric. This assumption further underscores what was stated in $\S 1$, i.e., the analysis herein is in most cases restricted to SISO, MISO, and diagonal form MIMO systems.

Consequently, let us assume $A\left(q^{-1}\right)$ to be symmetric (in the sense that matrices $A_{1}, \ldots, A_{n_{A}}$ are all symmetric). Without much loss of generality we can now assume the system to be SISO. (61) and (62) thus take the form

$$
\begin{aligned}
& F_{1}=-\left[E \psi_{t} \psi_{t}^{\mathrm{T}}\right]^{-1} E \psi_{t} \varphi_{t}^{\mathrm{T}} \\
& F_{2}=-\left[E \varphi_{t} \varphi_{t}^{\mathrm{T}}\right]^{-1} E \varphi_{t} \psi_{t}^{\mathrm{T}}
\end{aligned}
$$

whereas (60) takes the form

$$
F=\left[E \psi_{t} \psi_{t}^{\mathrm{T}}\right]^{-1} E \psi_{t} \varphi_{t}^{\mathrm{T}}\left[E \varphi_{t} \varphi_{t}^{\mathrm{T}}\right]^{-1} E \varphi_{t} \psi_{t}^{\mathrm{T}}
$$

For the sake of simplicity we have now dropped all arguments of $\psi_{t}$ and $\varphi_{t}$.

The expression for $F$ given in (69) turns out to be very useful in the following. We note that $(\lambda, h)$ is an eigenvalue/eigenvector-pair of $F$ if and only if

$$
\left(\lambda E \psi_{t} \psi_{t}^{\mathrm{T}}-E \psi_{t} \varphi_{t}^{\mathrm{T}}\left[E \varphi_{t} \varphi_{t}^{\mathrm{T}}\right]^{-1} E \varphi_{t} \psi_{t}^{\mathrm{T}}\right) h=0
$$

Theorem 1

If $\lambda$ is an eigenvalue of $F$, then $\lambda$ is real and $0 \leqslant \lambda \leqslant 1$.

Proof. See the Appendix.

At this point we know that no eigenvalues are outside the unit circle, which is necessary for local convergence. Furthermore, since the eigenvalues are real and positive, the convergence will, provided the algorithm does converge, be smooth, i.e., without any oscillations. The following lemma gives a characterization of the multiplicity of the eigenvalue $\lambda=1$.

\section{Lemma $l$}

$\lambda=1$ is an eigenvalue of $F$ of multiplicity $p$ (both algebraic and geometric) if and only if $\mu=0$ is an eigenvalue of

$$
\left[\begin{array}{ll}
E \psi_{t} \psi_{t}^{\mathrm{T}} & E \psi_{t} \varphi_{t}^{\mathrm{T}} \\
E \varphi_{t} \psi_{t}^{\mathrm{T}} & E \varphi_{t} \varphi_{t}^{\mathrm{T}}
\end{array}\right]
$$


of multiplicity $p$.

Proof. See the Appendix.

The previous lemma is helpful because it allows us to determine the multiplicity of the eigenvalue $\lambda=1$ simply by finding the rank of the matrix (71). In order to continue this line of approach, consider the three polynomials

$$
\begin{aligned}
& C(z)=c_{0}+c_{1} z+\ldots+c_{n} z^{n} \\
& D(z)=d_{0}+d_{1} z+\ldots+d_{m} z^{m} \\
& E(z)=e_{0}+e_{1} z+\ldots+e_{l} z^{l}
\end{aligned}
$$

With $C(z), D(z)$, and $E(z)$ we associate the matrix (missing entries are all equal to zero)

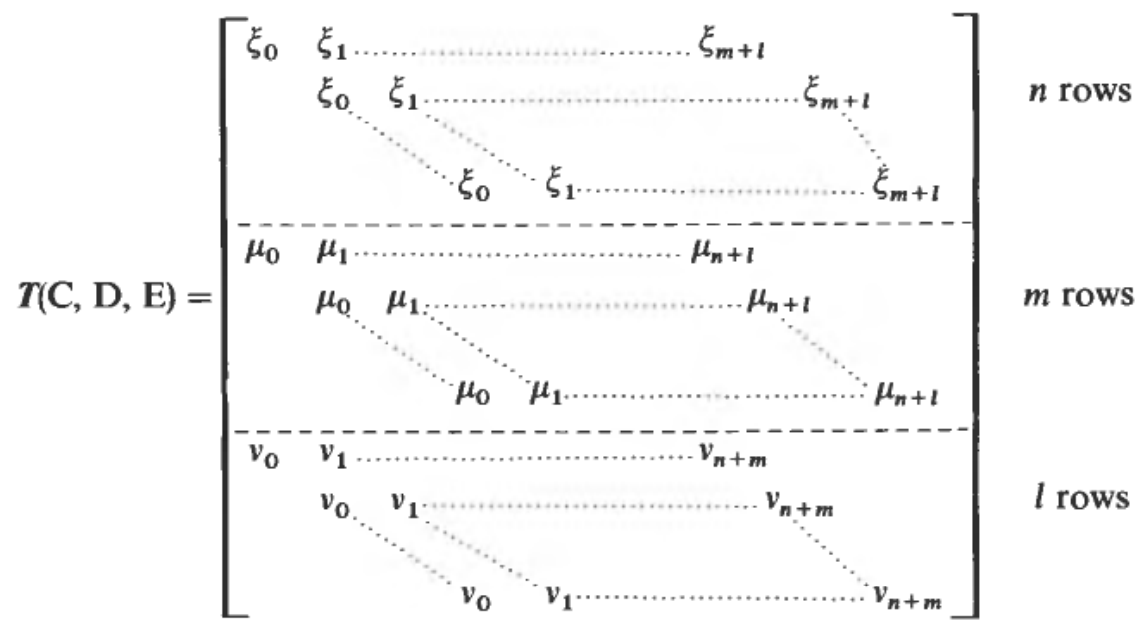

where

$$
\begin{aligned}
& \Xi(z)=D(z) E(z)=\xi_{0}+\xi_{1} z+\ldots+\xi_{m+l} z^{m+l} \\
& M(z)=C(z) E(z)=\mu_{0}+\mu_{1} z+\ldots+\mu_{n+l} z^{n+l} \\
& N(z)=C(z) D(z)=v_{0}+v_{1} z+\ldots+v_{n+m} z^{n+m}
\end{aligned}
$$

Matrix T(C, D, E) resembles a Sylvester matrix, see Jury (1974), Anderson and Jury (1976), Kailath (1980), and Söderström and Stocia (1983), and it can in fact be considered to be a kind of generalization. For example, if we choose $E(z)=1$, then $\mathbf{T}(\mathrm{C}$, $\mathrm{D}, \mathrm{E})=\mathbf{S}(\mathrm{D}, \mathrm{C})$ where $\mathrm{S}(\mathrm{D}, \mathrm{C})$ is an $(n+m) \times(n+m)$ Sylvester matrix.

\section{Lemma 2}

Assume $E(z)$ and $N(z)=C(z) D(z)$ to be coprime. Then

$$
\operatorname{rank} \mathbf{T}(\mathrm{C}, \mathrm{D}, \mathrm{E})=n+m+l-k
$$

if and only if $C(z)$ and $D(z)$ have exactly $k$ common zeros.

Proof. See the Appendix.

Use of the two lemmas is very helpful in proving the next theorem. 


\section{Theorem 2}

Assume the input $u_{t}$ is persistently exciting of order $n_{1}+n_{2}+n_{B}$. Then $\lambda=1$ is an eigenvalue of multiplicity $p$ if and only if $A_{1}\left(q^{-1}\right)$ and $A_{2}\left(q^{-1}\right)$ have exactly $p$ common zeros.

Proof. See the Appendix.

\section{Corollary 1}

The bootstrap DLS estimator converges locally if and only if $A_{1}\left(q^{-1}\right)$ and $A_{2}\left(q^{-1}\right)$ are coprime.

The results given by Theorem 1 and Theorem 2 are quite neat, since it is fairly easy to find when the bootstrap algorithm will fail. Multiple poles of the system must either belong solely to model $M_{1}$ or to model $M_{2}$ and may not be shared in any way.

Before we proceed with a couple of examples, we shall take a look at the two DIV estimators. In lieu of our previous comments we will now carry out the analysis only for SISO systems.

\subsection{Analysis of the DIVI Estimator}

As $N \rightarrow \infty$ the equations for the bootstrap DIV1 estimator take the form

$$
\begin{aligned}
& \hat{\beta}^{k+1}=\left[E \tilde{\psi}_{t}\left(\hat{\beta}^{k}, \hat{\theta}^{k}\right) \psi_{t}^{\mathrm{T}}\left(\hat{\theta}^{k}\right)\right]^{-1} E \tilde{\psi}_{t}\left(\hat{\beta}^{k}, \hat{\theta}^{k}\right)\left[w_{t}\left(\hat{\theta}^{k}\right)-B\left(q^{-1}, \hat{\theta}^{k}\right) u_{t}\right] \\
& \hat{\theta}^{k+1}=\left[E \tilde{\varphi}_{t}\left(\hat{\theta}^{k}\right) \varphi_{t}^{\mathrm{T}}\left(\hat{\beta}^{k+1}\right)\right]^{-1} E \tilde{\varphi}_{t}\left(\hat{\theta}^{k}\right) z_{t}\left(\hat{\beta}^{k+1}\right)
\end{aligned}
$$

By linearizing $(80)$ and $(81)$ about $\left(\beta^{*}, \theta^{*}\right)$ we obtain after calculations

$$
\begin{aligned}
& \hat{\beta}^{k+1}-\beta^{*}=F_{11}\left(\beta^{*}, \theta^{*}\right)\left[\hat{\beta}^{k}-\beta^{*}\right]+F_{12}\left(\beta^{*}, \theta^{*}\right)\left[\hat{\theta}^{k}-\theta^{*}\right] \\
& \hat{\theta}^{k+1}-\theta^{*}=F_{21}\left(\beta^{*}, \theta^{*}\right)\left[\hat{\theta}^{k}-\theta^{*}\right]+F_{22}\left(\beta^{*}, \theta^{*}\right)\left[\hat{\beta}^{k+1}-\beta^{*}\right]
\end{aligned}
$$

where

$$
\begin{aligned}
F_{11}\left(\beta^{*}, \theta^{*}\right)= & \tilde{M}^{-1}\left(\beta^{*}, \theta^{*}\right)\left\{E \frac{\partial \tilde{\psi}_{t}}{\partial \hat{\beta}^{\mathrm{T}}}\left(\beta^{*}, \theta^{*}\right)\right. \\
& \left.\times\left[w_{t}\left(\theta^{*}\right)-B\left(q^{-1}, \theta^{*}\right) u_{t}-\psi_{t}^{\mathrm{T}}\left(\theta^{*}\right) \beta^{*}\right]\right\} \\
F_{12}\left(\beta^{*}, \theta^{*}\right)= & \tilde{M}^{-1}\left(\beta^{*}, \theta^{*}\right)\left\{E \frac{\partial \tilde{\psi}_{t}}{\partial \hat{\theta}^{\mathrm{T}}}\left(\beta^{*}, \theta^{*}\right)\left[w_{t}\left(\theta^{*}\right)-B\left(q^{-1}, \theta^{*}\right) u_{t}-\psi_{t}^{\mathrm{T}}\left(\theta^{*}\right) \beta^{*}\right]\right. \\
& \left.+E \tilde{\psi}_{t}\left(\beta^{*}, \theta^{*}\right)\left[\frac{\partial w_{t}}{\partial \hat{\theta}^{\mathrm{T}}}\left(\theta^{*}\right)-\frac{\partial B}{\partial \hat{\theta}^{\mathrm{T}}}\left(q^{-1}, \theta^{*}\right) u_{t}-\beta^{* \mathrm{~T}} \frac{\partial \psi_{t}}{\partial \hat{\theta}^{\mathrm{T}}}\left(\theta^{*}\right)\right]\right\} \\
F_{21}\left(\beta^{*}, \theta^{*}\right)= & \tilde{L}^{-1}\left(\beta^{*}, \theta^{*}\right)\left\{E \frac{\partial \tilde{\varphi}_{t}}{\partial \hat{\theta}^{\mathrm{T}}}\left(\theta^{*}\right)\left[z_{t}\left(\beta^{*}\right)-\varphi_{t}^{\mathrm{T}}\left(\beta^{*}\right) \theta^{*}\right]\right\} \\
F_{22}\left(\beta^{*}, \theta^{*}\right)= & \tilde{L}^{-1}\left(\beta^{*}, \theta^{*}\right)\left\{E \tilde { \varphi } _ { t } ( \theta ^ { * } ) \left[\frac{\partial z_{t}}{\partial \hat{\beta}^{\mathrm{T}}}\left(\beta^{*}\right)-\theta^{* \mathrm{~T}} \frac{\partial \varphi_{t}}{\left.\left.\partial \hat{\beta}^{\mathrm{T}}\left(\beta^{*}\right)\right]\right\}}\right.\right.
\end{aligned}
$$

and where

$$
\begin{aligned}
\tilde{M}\left(\beta^{*}, \theta^{*}\right) & =E \tilde{\psi} \tilde{\psi}_{t}\left(\beta^{*}, \theta^{*}\right) \psi_{t}^{\mathrm{T}}\left(\theta^{*}\right) \\
\tilde{L}\left(\beta^{*}, \theta^{*}\right) & =E \tilde{\varphi}_{t}\left(\theta^{*}\right) \varphi_{t}^{\mathrm{T}}\left(\beta^{*}\right)
\end{aligned}
$$


Substituting from (82) into (83) we obtain (all arguments are now dropped for the sake of simplicity)

$$
\hat{\theta}^{k+l}-\theta^{*}=F_{22} F_{11}\left[\hat{\beta}^{k}-\beta^{*}\right]+\left(F_{21}+F_{22} F_{12}\right)\left[\hat{\theta}^{k}-\theta^{*}\right]
$$

which together with (82) shows that the bootstrap estimator converges locally, provided the matrix

$$
\left[\begin{array}{cc}
F_{11} & F_{12} \\
F_{22} F_{11} & F_{21}+F_{22} F_{12}
\end{array}\right]
$$

has all of its eigenvalues inside the unit circle.

From further calculations we find

$$
F_{11}=0 ; \quad F_{21}=0
$$

so that the nonzero eigenvalues of the matrix (91) can be determined from $F_{22} F_{12}$ or, eqivalently, from $F_{12} F_{22}$. We choose to use the latter in the sequel. Proceeding even further, we end up with

$$
\begin{aligned}
\tilde{M}\left(\beta^{*}, \theta^{*}\right) & =E \tilde{\psi}_{t} \psi_{t}^{\mathrm{T}}=E \tilde{\psi}_{t} \tilde{\psi}_{t}^{\mathrm{T}} \\
\tilde{L}\left(\beta^{*}, \theta^{*}\right) & =E \tilde{\varphi}_{t} \varphi_{t}^{\mathrm{T}}=E \tilde{\varphi}_{t} \tilde{\varphi}_{t}^{\mathrm{T}}
\end{aligned}
$$

whereas

$$
\begin{aligned}
& F_{12}=-\left[E \tilde{\psi}_{t} \tilde{\psi}_{t}^{\mathrm{T}}\right]^{-1} E \tilde{\psi}_{t} \tilde{\varphi}_{t}^{\mathrm{T}} \\
& F_{22}=-\left[E \tilde{\varphi}_{t} \tilde{\varphi}_{t}^{\mathrm{T}}\right]^{-1} E \tilde{\varphi}_{t} \tilde{\psi}_{t}^{\mathrm{T}}
\end{aligned}
$$

and the algorithm converges locally if and only if the matrix

$$
\tilde{F}=F_{12} F_{22}=\left[E \tilde{\psi}_{t} \tilde{\psi}_{t}^{\mathrm{T}}\right]^{-1} E \tilde{\psi}_{t} \tilde{\varphi}_{t}^{\mathrm{T}}\left[E \tilde{\varphi}_{t} \tilde{\varphi}_{t}^{\mathrm{T}}\right]^{-1} E \tilde{\varphi}_{t} \tilde{\psi}_{t}^{\mathrm{T}}
$$

has all of its eigenvalues inside the unit circle.

Further analysis gives identical results to those obtained for the DSL estimator. We summarize the properties of the DIV1 estimator in what follows.

\section{Theorem 3}

If $\lambda$ is an eigenvalue of $\tilde{F}$, then $\lambda$ is real and $0 \leqslant \lambda \leqslant 1$. Moreover, if $u_{t}$ is persistently exciting of order $n_{1}+n_{2}+n_{B}$, then $\lambda=1$ is an eigenvalue of $\tilde{F}$ of multiplicity $p$ if and only if $A_{1}\left(q^{-1}\right)$ and $A_{2}\left(q^{-1}\right)$ have exactly $p$ common zeros.

Proof. Omitted, but it is very similar to the proof for the DLS estimator in the Appendix.

\section{Corollary 2}

The bootstrap DIV1 estimator converges locally if and only if $A_{1}\left(q^{-1}\right)$ and $A_{2}\left(q^{-1}\right)$ are coprime.

\subsection{Analysis of the DIV2 Estimator}

As $N \rightarrow \infty$ the equations for the bootstrap DIV2 estimator take the form

$$
\begin{aligned}
& \hat{\beta}^{k+1}=\left[E \tilde{\psi}_{t}\left(\hat{\beta}^{k}, \hat{\theta}^{k}\right) \psi_{t}^{\mathrm{T}}\right]^{-1} E \tilde{\psi}_{t}\left(\hat{\beta}^{k}, \hat{\theta}^{k}\right)\left[y_{t}-B\left(q^{-1}, \hat{\theta}^{k}\right) \tilde{u}_{t}\left(\hat{\theta}^{k}\right)\right] \\
& \hat{\theta}^{k+1}=\left[E \tilde{\varphi}_{t}\left(\hat{\beta}^{k+1}, \hat{\theta}^{k}\right) \varphi_{t}^{\mathrm{T}}\left(\hat{\beta}^{k+1}\right)\right]^{-1} E \tilde{\varphi}_{t}\left(\hat{\beta}^{k+1}, \hat{\theta}^{k}\right) y_{t}
\end{aligned}
$$


Linearizing (96) and (97) about $\left(\beta^{*}, \theta^{*}\right)$ yields two equations of the form given by (82)-(83), but where the matrices $F_{11}, F_{12}, F_{21}$, and $F_{22}$ now take the form

$$
\begin{aligned}
& F_{11}\left(\beta^{*}, \theta^{*}\right)=\tilde{M}^{-1}\left(\beta^{*}, \theta^{*}\right)\left\{E \frac{\partial \tilde{\psi}_{t}}{\partial \hat{\beta}^{\mathrm{T}}}\left(\beta^{*}, \theta^{*}\right)\right. \\
& \left.\times\left[y_{t}-B\left(q^{-1}, \theta^{*}\right) \tilde{u}_{t}\left(\theta^{*}\right)-\psi_{t}^{\mathrm{T}} \beta^{*}\right]\right\} \\
& F_{12}\left(\beta^{*}, \theta^{*}\right)=\tilde{M}^{-1}\left(\beta^{*}, \theta^{*}\right)\left\{E \frac{\partial \tilde{\psi}_{t}}{\partial \hat{\theta}^{\mathrm{T}}}\left(\beta^{*}, \theta^{*}\right)\right. \\
& \times\left[y_{t}-B\left(q^{-1}, \theta^{*}\right) \tilde{u}_{t}\left(\theta^{*}\right)-\psi_{t}^{\mathrm{T}} \beta^{*}\right]-E \tilde{\psi}_{t}\left(\beta^{*}, \theta^{*}\right) \\
& \left.\times\left[\frac{\partial B}{\partial \hat{\theta}^{\mathrm{T}}}\left(q^{-1}, \theta^{*}\right) \tilde{u}_{t}\left(\theta^{*}\right)+B\left(q^{-1}, \theta^{*}\right) \frac{\partial \tilde{u}_{t}}{\partial \hat{\theta}^{\mathrm{T}}}\left(\theta^{*}\right)\right]\right\} \\
& \left.F_{21}\left(\beta^{*}, \theta^{*}\right)=\tilde{L}^{-1}\left(\beta^{*}, \theta^{*}\right)\left\{E \frac{\partial \tilde{\varphi}_{t}}{\partial \hat{\theta}^{\mathrm{T}}}\left(\beta^{*}, \theta^{*}\right)\left[y_{t}-\varphi_{t}^{\mathrm{T}}\left(\beta^{*}\right) \theta^{*}\right)\right]\right\} \\
& F_{22}\left(\beta^{*}, \theta^{*}\right)=\tilde{L}^{-1}\left(\beta^{*}, \theta^{*}\right)\left\{E \frac{\partial \tilde{\varphi}_{t}}{\partial \tilde{\beta}^{\mathrm{T}}}\left(\beta^{*}, \theta^{*}\right)\left[y_{t}-\varphi_{t}^{\mathrm{T}}\left(\beta^{*}\right) \theta^{*}\right]\right. \\
& \left.-E \tilde{\varphi}_{t}\left(\beta^{*}, \theta^{*}\right) \theta^{* \mathrm{~T}} \frac{\partial \varphi_{t}}{\partial \tilde{\beta}^{\mathrm{T}}}\left(\beta^{*}\right)\right\}
\end{aligned}
$$

where

$$
\begin{aligned}
\tilde{M}\left(\beta^{*}, \theta^{*}\right) & =E \tilde{\psi}_{t}\left(\beta^{*}, \theta^{*}\right) \psi_{t}^{\mathrm{T}} \\
\tilde{L}\left(\beta^{*}, \theta^{*}\right) & =E \tilde{\varphi}_{t}\left(\beta^{*}, \theta^{*}\right) \varphi_{t}^{\mathrm{T}}\left(\beta^{*}\right)
\end{aligned}
$$

From the above equations it follows that the DIV2 boutstrap estimator converges locally provided the matrix given by $(91)$ (but now with the new matrices $F_{11}$, $F_{12}, F_{21}$, and $F_{22}$ defined by (98)-(101)) has all of its eigenvalues strictly inside the unit circle.

Further calculations reveal that also in this case

$$
\begin{aligned}
& F_{11}=0 ; \quad F_{21}=0 \\
& \tilde{M}\left(\beta^{*}, \theta^{*}\right)=E \tilde{\psi}_{t} \psi_{t}^{\mathrm{T}}=E \tilde{\psi}_{t} \tilde{\psi}_{t}^{\mathrm{T}} \\
& \tilde{L}\left(\beta^{*}, \theta^{*}\right)=E \tilde{\varphi}_{t} \varphi_{t}^{\mathrm{T}}=E \tilde{\varphi}_{t} \tilde{\varphi}_{t}^{\mathrm{T}}
\end{aligned}
$$

whereas

$$
\begin{aligned}
& F_{12}=-\left[E \tilde{\psi}_{t} \tilde{\psi}_{t}^{\mathrm{T}}\right]^{-1} E \tilde{\psi}_{t} \cdot \frac{A_{1}\left(q^{-1}\right)}{A_{2}\left(q^{-1}\right)} \tilde{\varphi}_{t}^{\mathrm{T}} \\
& F_{22}=-\left[E \tilde{\varphi}_{t} \tilde{\varphi}_{t}^{\mathrm{T}}\right]^{-1} E \tilde{\varphi}_{t} \cdot \frac{A_{2}\left(q^{-1}\right)}{A_{1}\left(q^{-1}\right)} \tilde{\psi}_{t}^{\mathrm{T}}
\end{aligned}
$$

The nonzero eigenvalues of the matrix (91) can therefore also in this case be determined from the matrix

$$
\tilde{F}=F_{12} F_{22}=\left[E \tilde{\psi}_{t} \tilde{\psi}_{t}^{\mathrm{T}}\right]^{-1} E \tilde{\psi}_{t} \cdot \frac{A_{1}\left(q^{-1}\right)}{A_{2}\left(q^{-1}\right)} \tilde{\varphi}_{1}^{T}\left[E \tilde{\varphi}_{t} \tilde{\varphi}_{t}^{\mathrm{T}}\right]^{-1} E \tilde{\varphi}_{t} \cdot \frac{A_{2}\left(q^{-1}\right)}{A_{1}\left(q^{-1}\right)} \tilde{\psi}_{t}
$$


Contrary to what was found for the DLS and the DIV1 estimators, analysis of (106) does not lead to any easy-to-check criterion which allows us to determine the local convergence of the DIV2 estimator, although it can be asserted that $\lambda=1$ will be an eigenvalue of $F$ if $A_{1}\left(q^{-1}\right)$ and $A_{2}\left(q^{-1}\right)$ are not coprime. However, each system has to be investigated separately, and local convergence will generally depend upon the true values of $\beta$ and $\theta$ as revealed by the analysis of a second-order system (see the next section). Even when $A_{1}\left(q^{-1}\right)$ and $A_{2}\left(q^{-1}\right)$ are comprime, the eigenvalues of $\tilde{F}$ are not confined to the interval $[0,1]$ but may actually be outside the unit disc. Furthermore, analysis of a second-order system reveals that when local convergence is ensured, the convergence rate will be quite poor. This estimator should therefore generally be avoided, since the DIV1 estimator turns out to have superior convergence properties.

\section{Some examples}

In this section we shall take a look at a few simple examples which will provide us with further insight into the convergence properties of the three decentralized estimators.

\section{Example 1 (Second-Order System)}

Consider a simple second-order system of the form

$$
\left(1+\beta^{*} q^{-1}\right)\left(1+\theta^{*} q^{-1}\right) y_{t}=u_{t}+v_{t}
$$

where $\left|\beta^{*}\right|<1,\left|\theta^{*}\right|<1$. Since the polynomial $B\left(q^{-1}\right)$ in this case is known, what we assume about the input becomes almost immaterial; $u_{t}$ may be white noise, deterministic, or even correlated with $v_{t}$.

\section{DLS Estimator}

For the DLS estimator to work properly we must assume $v_{t}$ to be white noise. Using (69) we find after some calculations

$$
F=\frac{\left(1-\beta^{* 2}\right)\left(1-\theta^{* 2}\right)}{\left(1-\beta^{*} \theta^{*}\right)^{2}}
$$

Since

$$
\left(1-\beta^{*} \theta^{*}\right)^{2}-\left(1-\beta^{* 2}\right)\left(1-\theta^{* 2}\right)=\left(\beta^{*}-\theta^{*}\right)^{2} \geqslant 0,
$$

it indeed follows that $0<F \leqslant 1$ and that $F=1$ if and only if $\beta^{*}=\theta^{*}$. Moreover, if $\left|\beta^{*}\right| \approx 1.0$ or $\left|\theta^{*}\right| \approx 1.0$ (but not both), then $F \approx 0$ which means that the convergence will be very fast, in fact perhaps so fast that only one or two iterations could be necessary.

\section{DIV1 Estimator}

The input $v_{t}$ may in this case be coloured. For the sake of simplicity $u_{t}$ is assumed to be white noise. Using (95) we find after some calculations

$$
\tilde{F}=\frac{\left(1-\beta^{* 2}\right)\left(1-\theta^{* 2}\right)}{\left(1-\beta^{*} \theta^{*}\right)^{2}}
$$


which is identical to that found for the DLS estimator. All the above results and comments therefore also apply to this estimator.

\section{2 Estimator}

With the same assumptions as above we now obtain using (106)

$$
\tilde{F}=\frac{\left[\beta^{*}\left(1-\beta^{*} \theta^{*}\right)^{2}-\theta^{*}\left(1-\beta^{* 2}\right)^{2}\right]\left[\beta^{*}\left(1-\theta^{* 2}\right)^{2}-\theta^{*}\left(1-\beta^{*} \theta^{*}\right)^{2}\right]}{\left[\beta^{*}\left(1-\theta^{* 2}\right)-\theta^{*}\left(1-\beta^{* 2}\right)\right]^{2}\left(1-\beta^{*} \theta^{*}\right)^{2}}
$$

Careful analysis of (110) reveals what follows. First, $\tilde{F}=1$ if $\beta^{*}=\theta^{*}$, which is the same as for the two other estimators. Second, $0<\tilde{F}<1$ if and only if $\beta^{*}$ and $\theta^{*}$ have identical signs, i.e., $\operatorname{sgn} \beta^{*}=\operatorname{sgn} \theta^{*}$, whereas $\tilde{F}>1$ if $\operatorname{sgn} \beta^{*} \neq \operatorname{sgn} \theta^{*}$. Moreover, if $\left|\theta^{*}\right| \approx 0$ then $\widetilde{F} \approx 1$ regardless of the value of $\beta^{*}$, i.e., there is very slow convergence even when the system is extremely stiff, the opposite of that found for the other two estimators. Finally, assume for example that $\beta^{*} \approx-1.0$ (but still inside the unit circle). (110) then takes the approximate form

$$
\tilde{F}=1+\frac{\theta^{*}}{\left(1-\theta^{*}\right)^{2}}
$$

which means that $0.75<\tilde{F}<1.0$ whenever the estimator is stable, i.e., whenever $-1.0<\theta^{*}<0$. The latter reveals that convergence of this estimator will in any case be rather slow.

This example shows that compared to the other two estimators, the DIV2 estimator has significantly more restricted convergence properties, and the convergence rate will in any case be quite poor. The DIV2 estimator should therefore generally be avoided, since the DIV1 estimator obviously has superior convergence properties.

\section{Example 2 (Third-Order System)}

Consider the system

$$
\left(1+\beta^{*} q^{-1}\right)\left(1+\theta_{1}^{*} q^{-1}\right)\left(1+\theta_{2}^{*} q^{-1}\right) y_{t}=u_{t}+v_{t}
$$

where $\left|\beta^{*}\right|<1,\left|\theta_{1}^{*}\right|<1$, and $\left|\theta_{2}^{*}\right|<1$. Since $B\left(q^{-1}\right)$ is also known in this case, what we assume about the input $u_{t}$ is somewhat immaterial.

\section{DLS Estimator}

Assuming $v_{\mathrm{t}}$ to be white noise, we obtain using (69)

$$
F=\frac{\left(1-\beta^{* 2}\right)\left(1-\theta_{1}^{*} \theta_{2}^{*}\right)\left[\left(1+\beta^{* 2}\right)\left(1+\theta_{1}^{*} \theta_{2}^{*}\right)-2 \beta^{*}\left(\theta_{1}^{*}+\theta_{2}^{*}\right)\right]}{\left(1-\beta^{*} \theta_{1}^{*}\right)^{2}\left(1-\beta^{*} \theta_{2}^{*}\right)^{2}}
$$

From (112) it can be confirmed that $F=1$ if and only if $\beta^{*}=\theta_{1}^{*}$ or $\beta^{*}=\theta_{2}^{*}$, whereas $0<F<1$ provided $\beta^{*} \neq \theta_{1}^{*}$ and $\beta^{*} \neq \theta_{2}^{*}$. Moreover, if $\left|\beta^{*}\right| \approx 1.0$ or $\left|\theta_{1}^{*} \theta_{2}^{*}\right| \approx 1.0$ (but not both), then $F \approx 1$ which means very fast convergence. This 

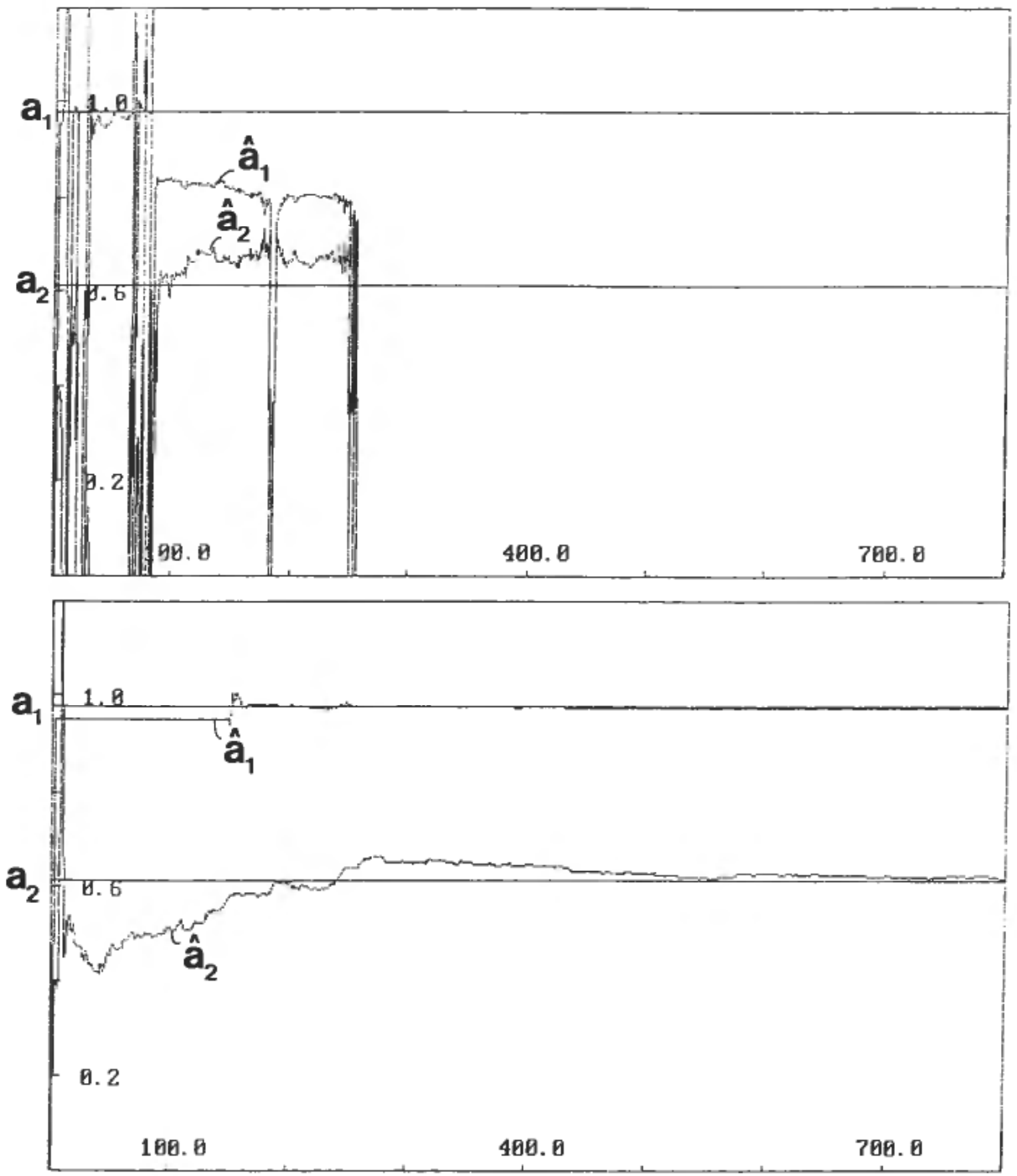

Figure 1. Estimates of the $A$-polynomial. (a) IV estimator; (b) DIV1 estimator.

confirms what was found in the first example, i.e., if the system is stiff, then the DLS estimator converges very rapidly.

Finally, assume $\theta_{1}^{*}<\beta^{*}<\theta_{2}^{*}$. Choosing for example $\beta^{*}=-0.5$ we now find $F \geqslant 0.75$ where the lower limit is reached by letting $\theta_{1}^{*} \rightarrow-1.0$ and $\theta_{2}^{*} \rightarrow 0$. This shows that the estimator can be quite sensitive to the choice of initial values of $\hat{\beta}$ and $\hat{\theta}$. Zeros of $A\left(q^{-1}\right)$ believed to be relatively close in order of magnitude should be put in the same factor of $A\left(q^{-1}\right)$, i.e., either in $A_{1}\left(q^{-1}\right)$ of in $A_{2}\left(q^{-1}\right)$. If in the latter case we had let $A_{1}\left(q^{-1}\right)=1+\theta_{1} q^{-1}$ (rather than $\left.A_{1}\left(q^{-1}\right)=1+\beta q^{-1}\right)$, a much better convergence rate would have been obtained.

From the above comments it is apparent that some information about the plant is not only helpful but in most cases also necessary for the estimator to work properly. Badly selected initial values or improper factoring of $A\left(q^{-1}\right)$ will in many cases cause the estimator to fail or to exhibit very slow convergence. 


\section{DIVI Estimator}

For the sake of simplicity, $u_{t}$ is again, as in Example 1, assumed to be white noise. Using (95) we find after some calculations

$$
\tilde{F}=\frac{\left(1-\beta^{* 2}\right)\left(1-\theta_{1}^{*} \theta_{2}^{*}\right)\left[\left(1+\beta^{* 2}\right)\left(1+\theta_{1}^{*} \theta_{2}^{*}\right)-2 \beta^{*}\left(\theta_{1}^{*}+\theta_{2}^{*}\right)\right]}{\left(1-\beta^{*} \theta_{1}^{*}\right)^{2}\left(1-\beta^{*} \theta_{1}^{*}\right)^{2}}
$$

which is identical to what was found for the DLS estimator. All the above results and comments therefore also apply to this estimator.

\section{Example 3 ( $A$ simulation experiment)}

Consider a moderately stiff system with transfer function $G(s)=1$ / $(1+0 \cdot 2 s)(1+5 s)$, which with a sampling period of $T=0 \cdot 1$ takes the form

$$
\left(1-0.98 q^{-1}\right)\left(1-0.61 q^{-1}\right) y_{t}=\left(0.0042 q^{-1}+0.0036 q^{-2}\right) u_{t}+v_{t}
$$

$\left\{u_{t}\right\}$ and $\left\{v_{t}\right\}$ are assumed to be mutually independent white processes. In the noisefree case $\left(v_{t} \equiv 0\right)$ an ordinary DLS estimator will perform somewhat better than the DIV1 estimator, but this is radically changed even with small disturbances. Results from a simulation experiment with a signal-to-noise ratio equal to 25 are shown in Fig. 1. Initial values for the estimators where chosen to be $\hat{a}_{1}^{0}=0.95, \hat{a}_{2}^{0}=0 \cdot 4, \hat{b}_{1}^{0}=$ 0.02 , and $\hat{b}_{2}^{0}=0.01$. The value of $\hat{a}_{1}$ was for the DIV1 estimator kept constant during a commissioning period of 150 samples.

As seen from Figs. 1 $(a)-1(b)$ and $2(a)-2(b)$, the ordinary IV estimator is completely unable to track the four parameters (the estimates of $a_{1}$ and $a_{2}$ are put equal to zero whenever they become complex). On the other hand, the DIV1 estimator tracks the four parameters reasonably well.

Similar results are reported in a comparison between an ordinary LS estimator and the DLS estimator in the paper by Young et al. (1987).

\section{Conclusion}

We have considered the problem of estimating an ill-natured, e.g., stiff, system by employing a certain decentralized estimation technique where each sub-estimator uses filtered input-output data to estimate a reduced form of the model. The decentralized estimator can be designed using LS or IV methods. We have also analysed the convergence properties of some different bootstrap estimators. As expected, not all of the estimators turned out to have good convergence properties. With a couple of estimators we have succeeded in finding necessary and sufficient conditions for local convergence. Some simple examples show that the estimators can exhibit very rapid convergence when the system is stiff.

The simulation experiment reported in this paper reveals that the robustness of the estimator can be significantly improved by employing a decentralized estimation scheme as presented. Furthermore, since the decentralized algorithms reduce the full estimation problem to two or more parallel estimators of smaller dimension, the computational load and memory requirements can be reduced.

It is believed that the inclusion of a priori knowledge of the plant is helpful and in many cases necessary in order to enable a decentralized estimator to work prop- 

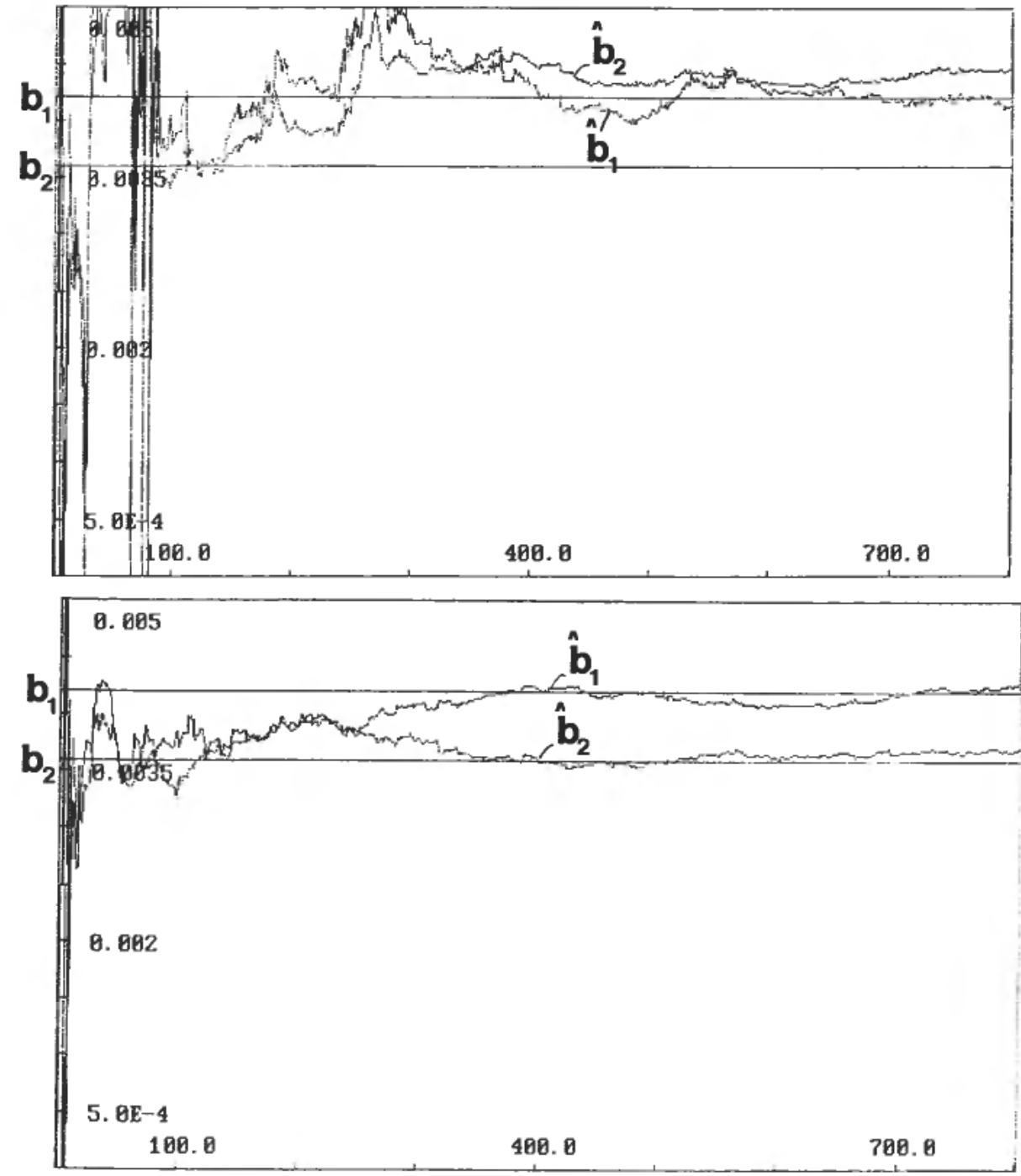

Figure 2. Estimates of the B-polynomial. (a) IV estimator; (b) DIV1 estimator.

erly. The examples fully demonstrate that improper factoring of $A\left(q^{-1}\right)$ can lead to very poor convergence properties.

We have not considered the case of factoring the polynomial $B\left(q^{-1}\right)$ in addition to factoring $A\left(q^{-1}\right)$, e.g., we can rewrite the model as

$$
A_{1}\left(q^{-1}\right) A_{2}\left(q^{-1}\right) y_{t}=B_{1}\left(q^{-1}\right) B_{2}\left(q^{-1}\right) u_{t}+v_{t}
$$

Defining

$$
w_{t}=A_{2}\left(q^{-1}\right) y_{t} ; \quad r_{t}=B_{2}\left(q^{-1}\right) u_{t},
$$

(110) takes the form

$$
A_{1}\left(q^{-1}\right) w_{t}=B_{1}\left(q^{-1}\right) r_{t}+v_{t}
$$

which can be used for estimating $A_{1}\left(q^{-1}\right)$ and $B_{1}\left(q^{-1}\right)$. Note that we here have to filter both the output $y_{t}$ and the input $u_{t}$. The polynomials $A_{2}\left(q^{-1}\right)$ and $B_{2}\left(q^{-1}\right)$ can 
be estimated in a similar manner. This estimation scheme requires that also the polynomials $B_{1}\left(q^{-1}\right)$ and $B_{2}\left(q^{-1}\right)$ must be coprime.

An important topic which has not been examined in this paper is the accuracy of the decentralized estimators. This will be an important topic for future research, and it is hoped that some results for this can be presented in the near future.

\section{REFERENCES}

Anderson, B. D. O., and JURY, E. I. (1976). Generalized Bezoutian and Sylvester matrices in multivariable linear control. IEEE Trans. on Automatic Control, 21, 551-556.

Clary, J. P. and Franklin, G. F. (1984). Self-turning control with a priori plant knowledge. Proc. 23rd IEEE Conference on Decision and Control, Las Vegas, Nevada, pp. 369-374.

HeNRIKSEN, R. (1988). Convergence analysis of some decentralized parameter estimators. Proc. 8th IF AC/IFORS Symposium on Identification and System Parameter Estimation, Beijing, China, pp. 447-452.

JURY, E. I. (1974). Inners and Stability of Dynamic Systems. (John Wiley \& Sons.) New York.

KaIlath, T. (1980). Linear Systems (Prentice-Hall) Englewood Cliffs, N.J.

SöDerström, T. and Stoica, P. G. (1983). Instrumental Variable Methods for System Identification (Springer-Verlag).

Stoica, P. G. and SöDerströ̀, T. (1981). Asymptotic behaviour of some bootstrap estimators. International Journal of Control, 33, 433-454.

VeTter, W. J. (1970). Derivative operations on matrices. IEEE Trans. on Automatic Control, $15,241-244$.

VETTER, W. J. (1973). Matrix calculus operations and Taylor expansions. SIAM Review, 15, $352-369$.

Young, E. E., Henriksen, R. and Mellichamp, D. A. (1987). A multi-rate decentralized parameter estimation method for stiff systems. Proc. 26th IEEE Conference on Decision and Control, Los Angeles, California, pp. 1902-1907.

\section{Appendix}

\section{Proof of Theorem 1}

Let $(\lambda, h)$ satisfy $(70)$, i.e.,

$$
\lambda E \psi_{t} \psi_{t}^{\mathrm{T}} h=E \psi_{t} \varphi_{t}^{\mathrm{T}}\left[E \varphi_{t} \varphi_{t}^{\mathrm{T}}\right]^{-1} E \varphi_{t} \psi_{t}^{\mathrm{T}}
$$

Multiplying both sides of (A1) by $h^{*}\left(h^{*}\right.$ denotes the adjoint, i.e., transpose and complex conjugate of $h$ ) we obtain

$$
\lambda h^{*} E \psi_{t} \psi_{t}^{\mathrm{T}} h=h^{*} E \psi_{t} \varphi_{t}^{\mathrm{T}}\left[E \varphi_{t} \varphi_{t}^{\mathrm{T}}\right]^{-1} E \varphi_{t} \psi_{t}^{\mathrm{T}} h
$$

Since the matrices $E \psi_{t} \psi_{t}^{\mathrm{T}}$ and $E \psi_{t} \varphi_{t}^{\mathrm{T}}\left[E \varphi_{t} \varphi_{t}^{\mathrm{T}}\right]^{-1} E \varphi_{t} \psi_{t}^{\mathrm{T}}$ are positive and nonnegative respectively, the term $h^{*} E \psi_{t} \psi_{t}^{\mathrm{T}} h$ must be real and positive, whereas $h^{*} E \psi_{t} \varphi_{t}^{\mathrm{T}}\left[E \varphi_{t} \varphi_{t}^{\mathrm{T}}\right]^{-1} E \varphi_{t} \psi_{t}^{\mathrm{T}} h$ must be real and non-negative. This implies that $\lambda$ must be real and $\lambda \geqslant 0$. Furthermore, $h$ must be real, i.e., $h^{*}=h^{\mathrm{T}}$ because $\lambda$ is real.

Now, since

$$
E\left[\begin{array}{c}
\psi_{t} \\
\varphi_{t}
\end{array}\right]\left[\psi_{t}^{\mathrm{T}} \varphi_{t}^{\mathrm{T}}\right]=\left[\begin{array}{cc}
E \psi_{t} \psi_{t}^{\mathrm{T}} & E \psi_{t} \varphi_{t}^{\mathrm{T}} \\
E \varphi_{t} \psi_{t}^{\mathrm{T}} & E \varphi_{t} \varphi_{t}^{\mathrm{T}}
\end{array}\right] \geqslant 0
$$

it follows that

$$
E \psi_{t} \psi_{t}^{\mathrm{T}} \geqslant E \psi_{t} \varphi_{t}^{\mathrm{T}}\left[E \varphi_{t} \varphi_{t}^{\mathrm{T}}\right]^{-1} E \varphi_{t} \psi_{t}^{\mathrm{T}}
$$


i.e.,

$$
h^{\mathrm{T}} E \psi_{t} \psi_{t}^{\mathrm{T}} h \geqslant h^{\mathrm{T}} E \psi_{t} \varphi_{t}^{\mathrm{T}}\left[E \varphi_{t} \varphi_{t}^{\mathrm{T}}\right]^{-1} E \varphi_{t} \psi_{t}^{\mathrm{T}} h
$$

which together with (A2) shows that $\lambda \leqslant 1$.

\section{Proof of Lemma 1}

Note that $(1, h)$ is an eigenvalue/eigenvector-pair of $F$ if and only if

$$
\left[E \psi_{t} \psi_{t}^{\mathrm{T}}-E \psi_{t} \varphi_{t}^{\mathrm{T}}\left[E \varphi_{t} \varphi_{t}^{\mathrm{T}}\right]^{-1} E \varphi_{t} \psi_{t}^{\mathrm{T}}\right] h=0
$$

First, assume that $\mu=0$ is an eigenvalue of the matrix (71) of multiplicity $p$. Since this matrix is symmetric, multiplicity $p$ means both algebraic and geometric multiplicity $p$. Let the vector $g^{\mathrm{T}}=\left(g_{1}^{\mathrm{T}}, g_{2}^{\mathrm{T}}\right) \neq 0$ satisfy

$$
\left[\begin{array}{cc}
E \psi_{t} \psi_{t}^{\mathrm{T}} & E \psi_{t} \varphi_{t}^{\mathrm{T}} \\
E \varphi_{t} \psi_{t}^{\mathrm{T}} & E \varphi_{t} \varphi_{t}^{\mathrm{T}}
\end{array}\right]\left[\begin{array}{l}
g_{1} \\
g_{2}
\end{array}\right]=0
$$

from which we obtain

$$
g_{2}=-\left[E \varphi_{t} \varphi_{t}^{\mathrm{T}}\right]^{-1} E \varphi_{t} \psi_{t}^{\mathrm{T}} g_{1}
$$

and

$$
\left[E \psi_{t} \psi_{t}^{\mathrm{T}}-E \psi_{t} \varphi_{t}^{\mathrm{T}}\left[E \varphi_{t} \varphi_{t}^{\mathrm{T}}\right]^{-1} E \varphi_{t} \psi_{t}^{\mathrm{T}}\right] g_{1}=0
$$

(A8) shows that $\left(1, g_{1}\right)$ must be an eigenvalue/eigenvector-pair of $F$. Furthermore, there are exactly $p$ distinct vectors $g$ satisfying (A7), which shows that $\lambda=1$ must have geometric multiplicity greater than or equal to $p$. In order to show that $\lambda=1$ cannot have algebraic (and hence geometric) multiplicity greater than $p$, we simply note that if $h$ is a vector satisfying (A6), then $(0, h)$ must be an eigenvalue/ eigenvector-pair of the symmetric matrix

$$
E \psi_{t} \psi_{t}^{\mathrm{T}}-E \psi_{t} \varphi_{t}^{\mathrm{T}}\left[E \varphi_{t} \varphi_{t}^{\mathrm{T}}\right]^{-1} E \varphi_{t} \psi_{t}^{\mathrm{T}}
$$

Therefore, if $\lambda=1$ is an eigenvalue of $F$ of algebraic multiplicity greater than $p$, it would also have to be of geometric multiplicity greater than $p$. This would, however, imply that $\mu=0$ must be an eigenvalue of the matrix (71) of multiplicity greater than $p$, contradicting the fact that $\mu=0$ is an eigenvalue of multiplicity $p$.

Second, assume $\lambda=1$ is an eigenvalue of $F$ of algebraic multiplicity $p$. From the above arguments it now follows easily that $\lambda=1$ also has geometric multiplicity $p$ and that $\mu=0$ must be an eigenvalue of the matrix (71) of multiplicity $p$.

\section{Proof of Lemma 2}

Consider the equation

$$
x^{\mathbf{T}} T(C, D, E)=0
$$

where

$$
x^{\mathrm{T}}=\left[\tilde{c}_{0}, \ldots, \tilde{c}_{n-1}, \tilde{d}_{0}, \ldots, \tilde{d}_{m-1}, \tilde{e}_{0}, \ldots, \tilde{e}_{l-1}\right]
$$


Define the polynomials

$$
\begin{aligned}
& \tilde{C}(z)=\tilde{c}_{0}+\tilde{c}_{1} z+\ldots+\tilde{c}_{n-1} z^{n-1} \\
& \tilde{D}(z)=\tilde{d}_{0}+\tilde{d}_{1} z+\ldots+\tilde{d}_{m-1} z^{m-1} \\
& \tilde{E}(z)=\tilde{e}_{0}+\tilde{e}_{1} z+\ldots+\tilde{e}_{l-1} z^{l-1}
\end{aligned}
$$

This allows us to write (A9) as

$$
\tilde{C}(z) D(z) E(z)+\tilde{D}(z) C(z) E(z)+\tilde{E}(z) C(z) D(z)=0
$$

Assume $C(z)$ and $D(z)$ to have exactly $k$ common zeros. Then we can write

$$
C(z)=C_{0}(z) L(z) ; \quad D(z)=D_{0}(z) L(z)
$$

where $\operatorname{deg} L(z)=k$ and where $C_{0}(z)$ and $D_{0}(z)$ are coprime. Substituting this into (A10), it takes the form

$$
\tilde{C}(z) D_{0}(z) E(z)+\tilde{D}(z) C_{0}(z) E(z)+\tilde{E}(z) C_{0}(z) D_{0}(z) L(z)=0
$$

Since $C_{0}(z), D_{0}(z)$, and $E(z)$ all are mutually coprime, it follows that $C_{0}(z)$ must be a divisor of $\tilde{C}(z)$, and $D_{0}(z)$ must be a divisor of $\tilde{D}(z)$, viz.

$$
\tilde{C}(z)=Q(z) C_{0}(z) ; \quad \tilde{D}(z)=R(z) D_{0}(z)
$$

where $\operatorname{deg} Q(z)=\operatorname{deg} R(z)=k-1$. Substituting (A13) into (A12) we obtain

$$
Q(z) E(z)+R(z) E(z)+\widetilde{E}(z) L(z)=0
$$

Since $E(z)$ and $L(z)$ are coprime $(L(z)$ is a divisor of both $C(z)$ and $D(z)$, i.e., of $C(z) D(z)), E(z)$ must be a divisor of $\tilde{E}(z)$. However, $\operatorname{deg} E(z)=l$, whereas $\operatorname{deg} \tilde{E}(z)=l$ -1 , and the only possibility is $\widetilde{E}(z)=0$. (A14) thus becomes

$$
Q(z)+R(z)=0
$$

where the polynomial

$$
Q(z)=q_{0}+q_{1} z+\ldots+={ }_{k-1} z^{k-1}
$$

has $k$ arbitrary coefficients, whereas $R(z)=-Q(z)$. This shows that the nullspace of $T^{\mathrm{T}}(C, D, E)$ must have dimension $k$, viz.

$$
\operatorname{dim} N\left(T^{\mathrm{T}}(C, D, E)\right)=k
$$

which means that rank $T^{\mathrm{T}}(C, D, E)=\operatorname{rank} T(C, D, E)=n+m+l-k$.

Finally, assume that rank $T(C, D, E)=n+m+l-k$. From the above arguments it is now easily derived that $C(z)$ and $D(z)$ must have exactly $k$ common zeros.

\section{Proof of Theorem 2}

We shall for the sake of simplicity assume $v_{t}=0$ for all $t$. The theorem is still generically $\dagger$ true for $v_{t} \neq 0$.

First, assume that $A_{1}\left(q^{-1}\right)$ and $A_{2}\left(q^{-1}\right)$ have exactly $p$ common zeros. Also, note that it was initially assumed that $A\left(q^{-1}\right)\left(=A_{1}\left(q^{-1}\right) A_{2}\left(q^{-1}\right)\right)$ and $B\left(q^{-1}\right)$ are

† Generically true here means true for almost all signal to noise ratios, see also Söderström and Stoica (1983). 
coprime. Now, observe that

$$
\begin{aligned}
& {\left[\begin{array}{l}
\Psi_{t} \\
\varphi_{t}
\end{array}\right]=} {\left[\begin{array}{c}
-\frac{B\left(q^{-1}\right)}{A_{1}\left(q^{-1}\right)} u_{t-1} \\
\vdots \\
-\frac{B\left(q^{-1}\right)}{A_{1}\left(q^{-1}\right)} u_{t-n_{1}} \\
-\frac{B\left(q^{-1}\right)}{A_{2}\left(q^{-1}\right)} u_{t-1} \\
\vdots \\
-\frac{B\left(q^{-1}\right)}{A_{2}\left(q^{-1}\right)} u_{t-n_{2}} \\
u_{t-1} \\
\vdots \\
u_{t-n_{B}}
\end{array}\right]=} \\
& \frac{1}{A_{1}\left(q^{-1}\right) A_{2}\left(q^{-1}\right)}\left[\begin{array}{c}
-A_{2}\left(q^{-1}\right) B\left(q^{-1}\right) u_{t-1} \\
\vdots \\
-A_{2}\left(q^{-1}\right) B\left(q^{-1}\right) u_{t-n_{1}} \\
A_{1}\left(q^{-1}\right) B\left(q^{-1}\right) u_{t-1} \\
\vdots \\
-A_{1}\left(q^{-1}\right) B\left(q^{-1}\right) u_{t-n_{2}} \\
A_{1}\left(q^{-1}\right) A_{2}\left(q^{-1}\right) u_{t-1} \\
\vdots \\
A_{1}\left(q^{-1}\right) A_{2}\left(q^{-1}\right) u_{t-n_{B}}
\end{array}\right]= \\
& \frac{\left.A_{1}, A_{2},-B\right)}{A_{1}\left(q^{-1}\right) A_{2}\left(q^{-1}\right)}\left[\begin{array}{l}
u_{t-1} \\
u_{t-2} \\
\vdots \\
u_{t-n_{1}-n_{2}-n_{B}}
\end{array}\right]
\end{aligned}
$$

where the matrix $T\left(A_{1}, A_{2},-B\right)$ is given by $(75)-(78)$. We now obtain

$$
\begin{aligned}
& E\left[\begin{array}{l}
\psi_{t} \\
\varphi_{t}
\end{array}\right]\left[\psi_{t}^{\mathrm{T}} \varphi_{t}^{\mathrm{T}}\right]=\left[\begin{array}{ll}
E \psi_{t} \psi_{t}^{\mathrm{T}} & E \psi_{t} \varphi_{t}^{\mathrm{T}} \\
E \varphi_{t} \psi_{t}^{\mathrm{T}} & E \varphi_{t} \varphi_{t}^{\mathrm{T}}
\end{array}\right]= \\
& T\left(A_{1}, A_{2},-B\right) P\left(A_{1} A_{2}, A_{1} A_{2}, u, n_{1}+n_{2}+n_{B}, n_{1}+n_{2}+n_{B}\right) T^{\mathrm{T}}\left(A_{1}, A_{2},-B\right)
\end{aligned}
$$

where

$$
P(C, D, u, n, m)=E\left\{\frac{1}{C\left(q^{-1}\right)}\left[\begin{array}{c}
u_{t-1} \\
\vdots \\
u_{t-n}
\end{array}\right] \cdot \frac{1}{D\left(q^{-1}\right)}\left[u_{t-1}, \ldots, u_{t-m}\right]\right\},
$$

see Söderström and Stoica (1983). If $C\left(q^{-1}\right)$ and $D\left(q^{-1}\right)$ are polynomials with all zeros strictly inside the unit circle and if $n \geqslant m$, then $\operatorname{rank} P(C, D, u, n, m)=m$ if either 
(1) $C\left(q^{-1}\right) / D\left(q^{-1}\right)$ is a strictly positive real function and $u_{t}$ is persistently exciting of order $m$

or

(2) The process $\left\{u_{t}\right\}$ is an ARMA $\left(n_{f}, n_{g}\right)$ process, i.e.,

$$
F\left(q^{-1}\right) u_{t}=G\left(q^{-1}\right) \varepsilon_{t}
$$

where $\operatorname{deg} F\left(q^{-1}\right)=n_{f}, \operatorname{deg} G\left(q^{-1}\right)=n_{g}$, and $\left\{\varepsilon_{t}\right\}$ is a white process.

In our case we have $A_{1}\left(q^{-1}\right) A_{2}\left(q^{-1}\right)=C\left(q^{-1}\right)=D\left(q^{-1}\right)$, so it is obviously sufficient that $u_{t}$ is persistently exciting of order $n_{1}+n_{2}+n_{B}$ for $P\left(A_{1} A_{2}, A_{1} A_{2}, u, n_{1}+n_{2}\right.$ $+n_{B}, n_{1}+n_{2}+n_{B}$ ) to have rank $n_{1}+n_{2}+n_{B}$. By the assumption being made in the theorem it thus follows that

$$
\operatorname{rank}\left[\begin{array}{cc}
E \psi_{t} \psi_{t} & E \psi_{t} \varphi_{t}^{\mathrm{T}} \\
E \varphi_{t} \psi_{t}^{\mathrm{T}} & E \varphi_{t} \varphi_{t}^{\mathrm{T}}
\end{array}\right]=\operatorname{rank} T\left(A_{1}, A_{2},-B\right)=n_{1}+n_{2}+n_{B}-p
$$

which means that the matrix (71) must have an eigenvalue $\mu=0$ of multiplicity $p$. From Lemma 1 it now follows that $F$ has an eigenvalue $\lambda=1$ of multiplicity $p$.

Second, assume $F$ to have an eigenvalue $\lambda=1$ of multiplicity $p$. By Lemma 1 $\mu=0$ must be an eigenvalue of the matrix (71) of multiplicity $p$. From (A17)-(A19) it then follows that rank $T\left(A_{1}, A_{2},-B\right)=n_{1}+n_{2}+n_{B}-p$, which by Lemma 2 implies that $A_{1}\left(q^{-1}\right)$ and $A_{2}\left(q^{-1}\right)$ must have exactly $p$ common zeros.

It is of interest to observe that this theorem can be proved in a somewhat more direct fashion using (69) and computing the power spectral density of $\left[\psi_{t}^{\mathrm{T}} \varphi_{t}^{\mathrm{T}}\right]^{\mathrm{T}}$. This proof is, however, quite lengthy and laborious. 